\title{
Design and Implementation of Inter-core Communication of Embedded Multiprocessor Based on Shared Memory
}

\author{
Yang Nie ${ }^{1,2}$, Lili Jing ${ }^{1}$ and Pengyu Zhao ${ }^{1}$ \\ ${ }^{1}$ Department of Physics, Jining Normal University, Inner Mongolia, China \\ ${ }^{2}$ Digital Engineering Center, Communication University of China, Beijing, China \\ nieyangwork@163.com
}

\begin{abstract}
In this paper, two Cortex-A9 processors and microblaze processor are used to build a multiprocessor embedded system. The whole system is connected AXI Interconnect, and the shared memory is used as a communication mechanism. Embedded multiprocessor system is mapped into FPGA, and the feasibility and practicability of the system are verified. Simulation result Indicate that shared memory provides very high performance and low latency access from both processors compared with the DDR memory.
\end{abstract}

Keywords: Inter-core Communication; Multiprocessor; Shared Memory, Zynq-7000

\section{Introduction}

With the rapid development of microelectronic technology, the development of embedded system has become increasingly mature[1]. Though Moore's law marches on, and the number of economically manufacturable transistors per chip continues increasing, clock frequencies have hit a wall because of power dissipation. It's gotten too hot to handle. Instead of increasing the clock frequency, designers can use larger transistor budgets to do more work per clock cycle. Within a single processor pipeline, techniques such as instruction-level parallelism, hardware threads, and data-parallel (SIMD) instructions have reached the point of diminishing returns.

It now makes more hardware sense to add multiple processor cores on chip and turn to task level parallelism. However, the performance of the multi-core processor is not simply increasing with the increase of the number of cores[2]. The performance of multi-core processor is given in many factors, such as the transmission mechanism, the design of the operating system, the software application development. Among all of the factors mentioned above, Inter-core communication mechanism is an important factor that affects the multi-core performance. Furthermore, the design of inter-core communication mechanism determines the communication overhead of the system and the shared resources of the system. On the other hand, the design of inter-core communication mechanism affects the length of the task scheduling, the parallelism and the throughput of the system.

The structure of this paper is organized as follows. Section 2 describes the basic structure of Zynq-7000, and the architecture of Cortex-A9 processor and microblaze processor are discussed in detail. Section 3 demonstrates the mechanism of communication, and the AXI Interconnect and shared memory are analyzed and studied. Two kinds of shared memory, on-chip local memory and on external memory, will be study. In Section 4, two Cortex-A9 processors and microblaze processor are used to construct a multi-core embedded system, and the inter-core 
communication mechanism is adopted by shared memory. Finally, concluding remarks are drawn in Section 5.

\section{The Cortex-A9 Processor of Zynq-7000 and MicroBlaze Processor}

The Zynq-7000 family is based on the Xilinx All Programmable SoC (AP SoC) architecture[3]. These products integrate a feature-rich dual-core ARM Cortex-A9 based processing system (PS) and Xilinx programmable logic (PL) in a single device. The PL section is ideal for implementing high-speed logic, arithmetic and data flow subsystems, while the PS supports software routines and/or operating systems, meaning that the overall functionality of any designed system can be appropriately partitioned between hardware and software.

The Zynq-7000 AP SoC provides two Cortex-A9 processors with maximum frequency of $667 \mathrm{MHz}$. The application processing unit (APU) consists of two ARM Cortex-A9 processor with a snoop control unit (SCU), which is responsible for maintaining the cache coherency between the two processors. The block diagram of simplified APU is shown in Figure 1. Each processor is a low-power and highperformance core. The CPU implements dynamic branch prediction and with its variable length pipeline can deliver 2.5 DMIPs/MHz. The Cortex-A9 processor implements the ARM v7-A architecture with full virtual memory support and can execute 32-bit ARM instructions, 16-bit and 32-bit Thumb instructions.

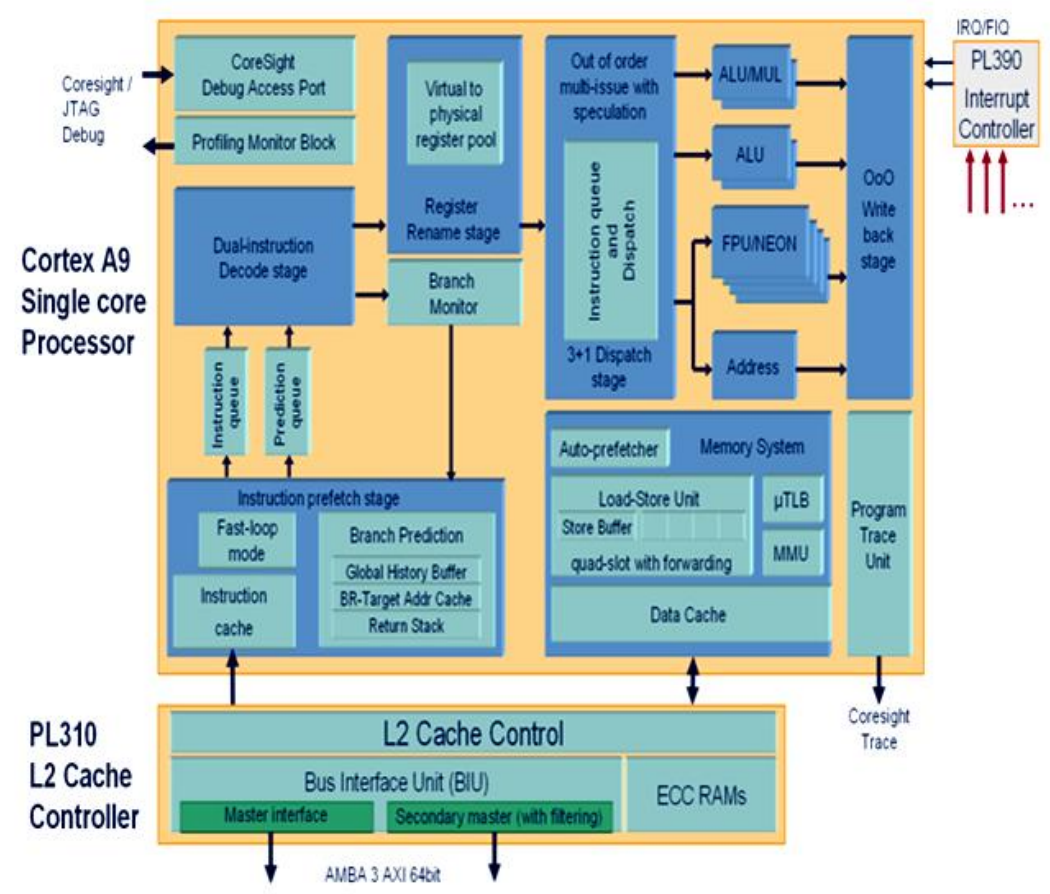

Figure 1. ARM Cortex-A9 Processor Micro-Architecture

Each processor has its own $32 \mathrm{~KB}$ level-one (L1) instruction and data caches, memory management unit (MMU), and separate media processing engine (NEON) [4]. L1 caches include two parts: instruction-side cache (I-Cache) and data-side cache (D-Cache). I-Cache is responsible for providing an instruction stream to the Cortex-A9 processor. D-Cache is responsible for holding the data used by the Cortex-A9 processor. The MMU in the ARM architecture involves both memory protection and address translation. The MMU works closely with the L1 and L2 memory systems in the process of translating virtual addresses to physical 
addresses. NEON is co-processor and extends the Cortex-A9 to provide support for the ARM v7 advanced single instruction multiple data (SIMD) and vector floatingpoint instruction sets.

The MicroBlaze is a 32-bit RISC machine that follows the classic RISC architecture previously described. It is a load/store machine with 32 general purpose registers[5]. All instructions are 32-bits wide and most execute in a single clock cycle. The block diagram of MicroBlaze basic structure is shown in Fig.2. The backbone of the architecture is a single-issue, 3-stage pipeline with 32 generalpurpose registers, an arithmetic logic unit (ALU), a shift unit, and two levels of interrupt. the processor includes 32-bit instruction word with three operands and two addressing modes and 32-bit address bus. The MicroBlaze soft core processor is highly configurable, allowing you to select a specific set of features required by your design.

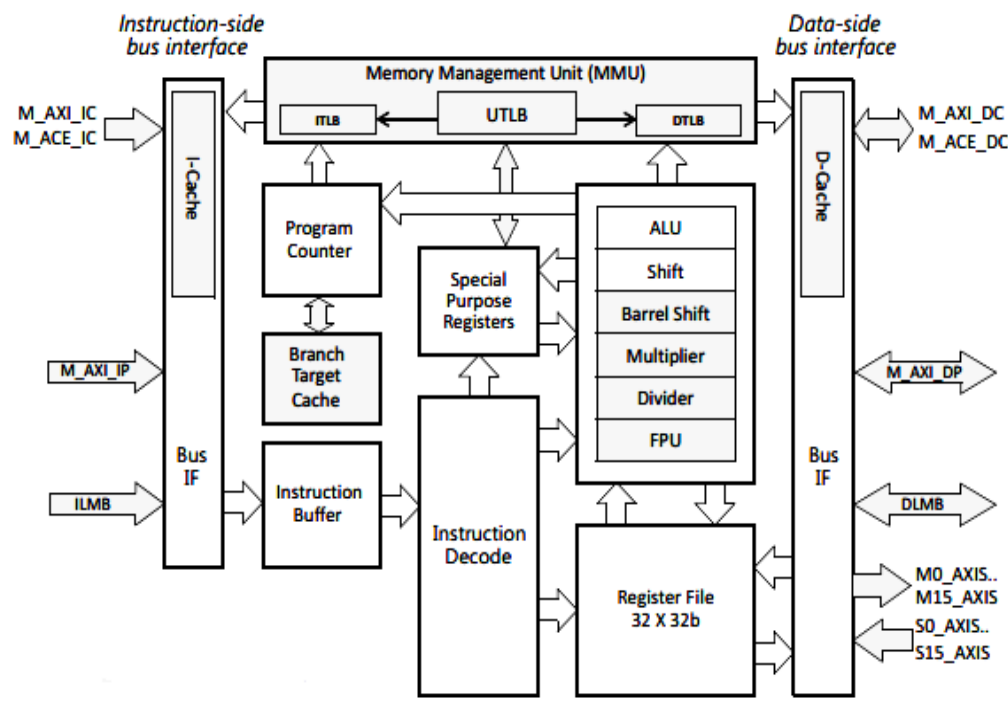

Figure 2. MicroBlaze Processor Block Diagram

The MicroBlaze core is organized as Harvard architecture with separate bus interface units for data and instruction accesses. The following four memory interfaces are supported: local memory bus (LMB), the AMBA AXI4 interface (AXI4), the IBM processor local bus (PLB), and Xilinx cache link (XCL). The LMB provides single-cycle access to on-chip dual port block RAM. The AXI4 and PLB interfaces provide a connection to both on-chip and off-chip peripherals and memory. The ACE interfaces provide cache coherent connections to memory. The cache link interface is intended for use with specialized external memory controllers. MicroBlaze also supports up to 16 fast simplex link (FSL) or AXI4stream interface ports, each with one master and one slave interface.

\section{The Mechanism of Inter-core Communication}

In this section, two kinds of communication mechanisms used in this design are introduced and studied. First, we will introduce the shared memory mechanism. Shared memory is used as inter-core communication mechanism in multi-core system. In addition, we will discuss the AXI 4, and it is a kind of high performance, high bandwidth, low latency on-chip bus. 


\subsection{Shared Memory}

Shared memory is the most common and intuitive way of passing information between processing subsystems. Because the mechanism has zero copy function, so it is very suitable for large data sharing. Shared memory is the fastest inter-process communication mechanism [6][7]. The operating system maps a memory segment in the address space of several processes, so that several processes can read and write in that memory segment without calling operating system functions. A shared memory system has the following properties:

1) Any processor can reference any shared memory location directly.

2) Communication occurs via processor load and store instructions.

3) Location of data in memory is transparent to the programmer. Data could be distributed across multiple processors, the details of which would then be abstracted away by some software API.

4) Access to the shared memory segment must be synchronized by some hardware/software protocol between the two processors.

Shared memory is typically the fastest asynchronous mode of communication, especially when the information to be shared is large. Shared memory also allows possible zero-copy or in-place message processing schemes. Shared memory can be built out of on-chip local memory or on external memory.

Sharing external memory is done using the Multi-Port Memory Controller (MPMC) memory controller[8] as shown in Figure 3. Each port of the memory controller is mapped to the same address range and though each port, all the processors automatically share the entire external memory. It is the responsibility of the software designer to define the distinct shared and non shared memory regions, based on some partitioning, and write the software protocol that uses the memory regions to pass information between the processing subsystems.

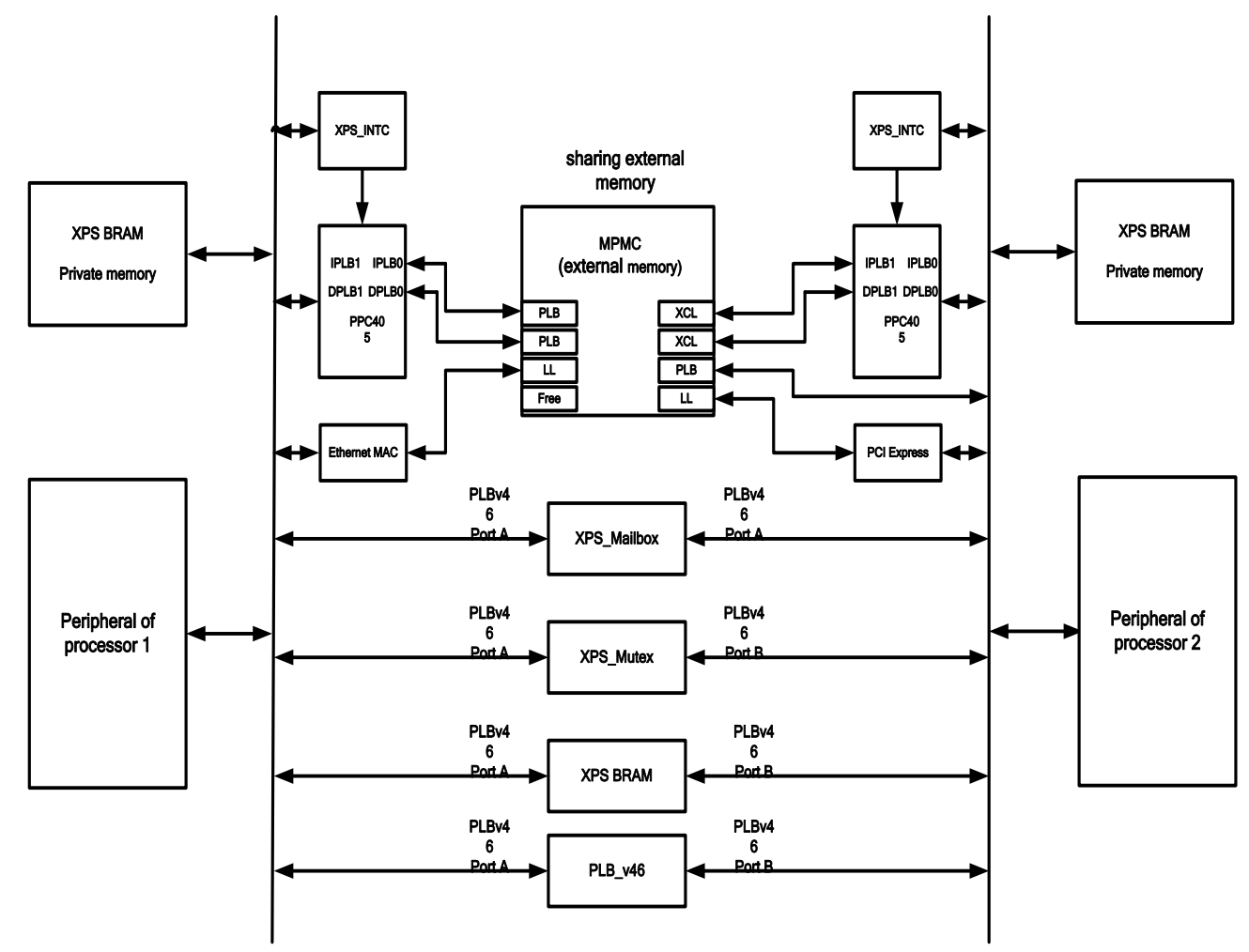

Figure 3. The External Shared Memory Based on MPMC 
On-chip memory can be shared using PLBv46 based memory controllers as shown in Figure 4. Apart from PLBv46, local memory interfaces such as OCM and LMB can also be used to create high-performance, guaranteed latency, shared memory segments. This scheme works by connecting memory controllers via local memory interfaces to the on-chip BRAM blocks. Because on-chip memory BRAM blocks on Xilinx FPGA are dual ported in nature, this scheme has the limitation that the memory can be shared between a maximum of two processors. The interfaces connected on either side can be LMB and/or OCM. This mode of sharing can be used between any pair of processors - two PowerPC processors, two MicroBlaze processors, or a PowerPC processor and a MicroBlaze processor as shown in Figure 4.

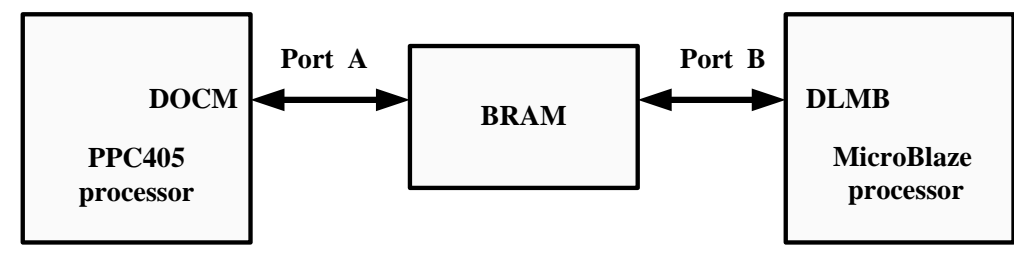

Figure 4. The on-chip Local Memory Based on BRAM

When using shared memory in conjunction with caches on the processors, the user must be aware of coherency considerations. Neither the MicroBlaze processors nor the PowerPC processors provide cache coherency support and the software must enforce coherency. This is described further the Software Design section. Note that using local memory interfaces to on-chip BRAM has the same performance as a cache hit, therefore such regions of memory are typically not marked as cacheable. Thus by using local memory for sharing, a multiprocessor system garners all the benefits guaranteed latency, performance, and memory coherency.

\subsection{AXI4 Interconnect}

AXI is a protocol belonging to the ARM AMBA family of microcontroller buses[9]. The AMBA protocol is an open standard on-chip interconnect specification, allowing the connection and management of many controllers and peripherals in a multi-master design. Much like the requirements for the rest of the AMBA family it is targeted at highperformance, high-frequency system designs. The AXI protocol is optimized for FPGA implementation. The fourth generation of AXI, known as AXI4, is the direct result of a partnership effort. AXI4 comes in three distinct flavors: AXI4, AXI4-Lite and AXI4Stream. AXI4 is a robust memory-mapped interface designed to achieve maximum levels of on-chip performance. Allows variable bursts up to 256 data transfers per single address transfer. AXI4-Lite is single-transaction memory-mapped interface. It is a smaller logic footprint, a subset of the AXI4 interface, used for accessing control registers and lowperformance peripherals. AXI4-Stream is used for high-speed streaming applications that do not require an address.

AXI4 interface connects one master to one slave device. While traditional interfaces specify a bus-style shared data path for communication, AXI4 provides greater communication flexibility by defining the interface as point to point. The AXI4 interface consists of five separate channels, as shown in Figure 5. 


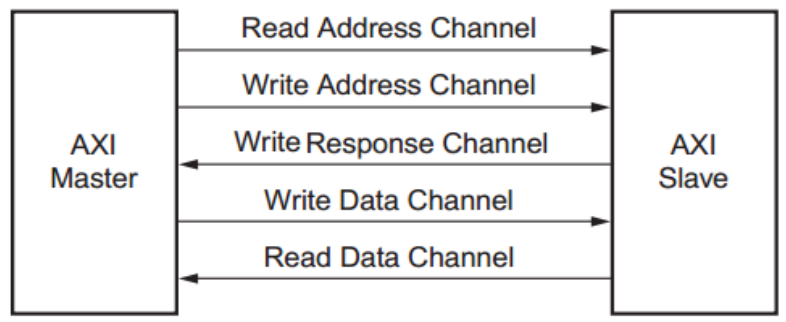

Figure 5. AXI4 Master-Slave Channel Architecture

Data can move in both directions between the master and slave simultaneously, and data burst lengths can vary up to 256 data transfers. For an AXI4 Read transaction, the master device issues information to the slave indicating the source location and data burst length. AXI4 Write cycles operate in a similar fashion, using a separate set of Write Address and Write Data channels. An AXI4 Write Response channel is used to communicate completion information so that write transfers can be pipelined and tracked throughout the system to improve data throughput.

\section{The Design of Multi-core Embedded System}

The design includes the hardware and software necessary to build a system that runs one Cortex-A9 processor and one MicroBlaze processor in an Asymmetric multiprocessing (AMP) configuration. AMP is a mechanism that allows multiple processors to run their own operating systems or applications. The second Cortex-A9 processor (CPU1) is not used in this design. The Cortex-A9 processor (CPU0) and MicroBlaze processor (MB0) are configured to run their own applications. Each CPU runs a application within its own standalone environment. OCM is used by both processors to communicate to each other. When compared to DDR memory, OCM provides very high performance and low latency access from both processors. the baremetal application running on CPU0 is the master of the system and is responsible for:

1) System initialization

2) Releasing PL reset

3) Communicating with MB0

4) Sharing the UART with MB0

The bare-metal application running on MB0 is responsible for:

1) Communicating with CPUO

2) Servicing interrupts from a core in the PL

3) Sharing the UART with CPU0

The Zynq SoC PS includes resources that are private to CPU0 and other resources that are accessible from MB0. some of the private resources include L1 cache, private peripheral interrupts (PPI), memory management unit (MMU) and private timers. some of the shared resources are interrupt control distributor (ICD), OCM, global timer, snoop control unit (SCU) and L2 cache and UART0.

In this design, CPU0 is treated as the master and controls the shared resources. If MB0 were to require control of a shared resource, it would have to communicate the request to CPU0 and let CPU0 control the resource. To avoid shared resource conflicts, the following measures have been adopted: 
1) CPU0 has only been made aware of memory at 0x00100000 to 0x2FFFFFFF. MB0 uses DDR memory from 0x30000000 to 0x3FFFFFFF for its own application.

2) Accesses to OCM are handled very carefully by each CPU to prevent contention. A single OCM address location is used as a flag to communicate between the two processors. CPU0 initializes the flag to 0 before starting MB0. When the flag is zero CPU0 owns the UART. When the flag is not zero, MB0 owns the UART. Only CPU0 sets the flag and only MB0 clears it.

\subsection{The Hardware Design of Multi-core Systems}

The hardware design of the system is realized in the PL section of the Zynq-7000. The PL block contains the MicroBlaze processor and a custom, embedded core connected to a synchronous output of a ChipScope analyzer VIO core [10][11]. The VIO core provides a mechanism for a user to interact with hardware from the ChipScope analyzer. The PL block diagram is shown in Fig.6. The core is also connected to MB0's data port (M_AXI_DP) through an AXI Interconnect that allows MB0 access to the control register within the core. MB0 accesses the control register to clear the interrupt request (IRQ) during the interrupt service routine. CPU0 can optionally use the control register to create an interrupt towards MB0. Any instruction or data fetches to the address range of 0x30000000 to 0x3FFFFFFF are forwarded to the PS HP0 and HP1 ports from the MicroBlaze processor's M_AXI instruction and cache ports. Any accesses outside of that range are forwarded through the MicroBlaze processor's M_AXI_DP data port.

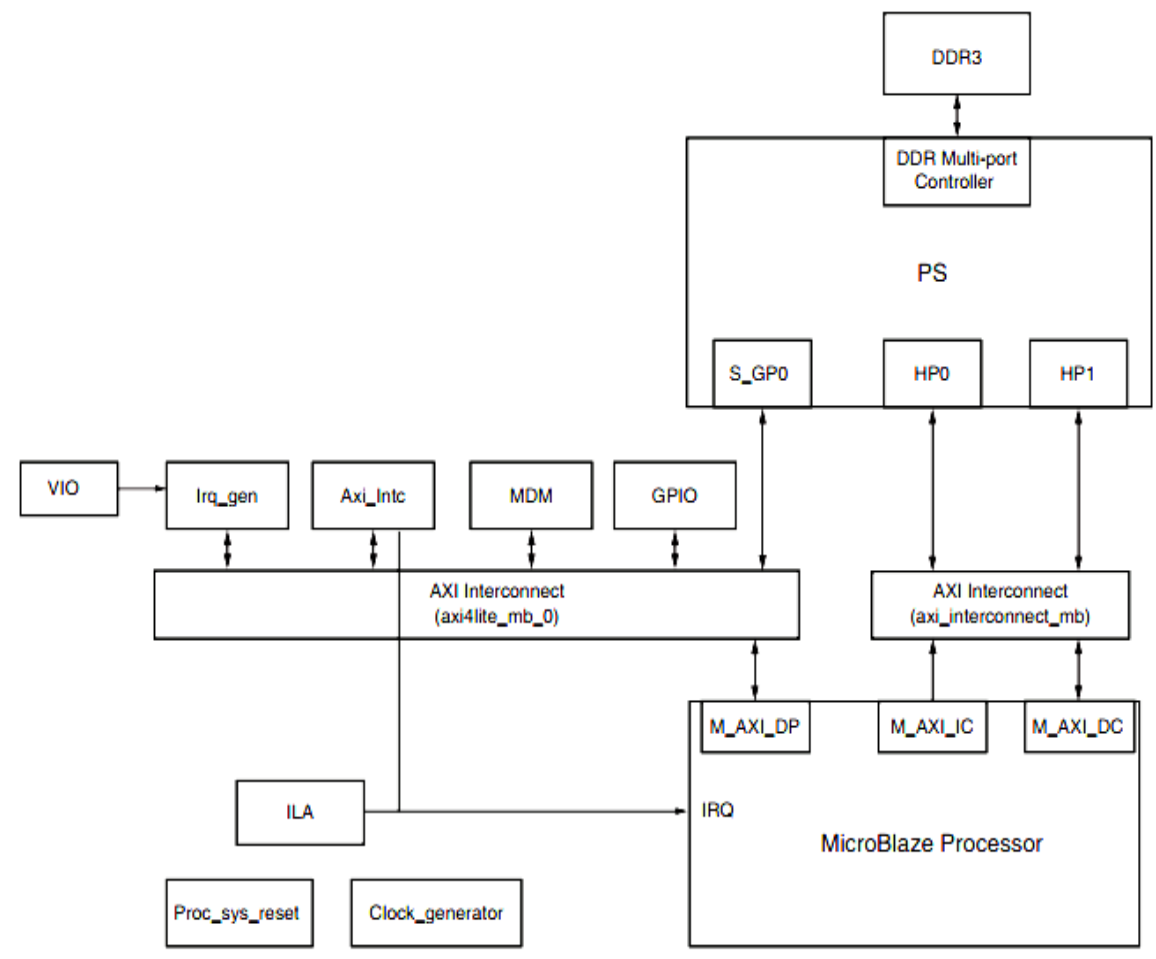

Figure 6. The Block Diagram of Part of PL

\subsection{The Software Design of Multi-core Systems}

The software can be broken down into three sections: First stage boot loader (FSBL), the own application for CPU0 and the own application for MB0 [12, 13].

The FSBL always runs on CPU0 and is the first software application that is run after power-on reset of the PS. The FSBL is responsible for programming the PL and copies 
both application executable and linkable format (ELF) files to DDR memory. After loading the applications to DDR memory, the FSBL then starts executing the first application that was loaded.

CPU0's application is located in memory starting at address 0x00100000. The linker script is used to set the starting address. The CPU0 application does the following:

1) Remaps the full $256 \mathrm{~KB}$ of OCM to the top of the address map (0xFFFC0000 to 0xFFFFFFFF) and disables DDR memory filtering which enables DDR memory at the bottom of CPU0's address map.

2) Configures the MMU to disable cache for OCM accesses in the address range of 0xFFFC0000 to 0xFFFFFFFF.

3) Releases the PL reset.

4) Prints "CPU0: Hello World" to the UART

5) Sets a memory location in OCM that is used as a semaphore flag.

6) Waits for the memory location in OCM that is used as a semaphore flag to be cleared.

After the PS powers up and the internal boot ROM completes execution, CPU1 is redirected to a small piece of code in OCM at 0xFFFFFE00. This piece of code is a continuous loop that waits for an event, checks address location 0xFFFFFFF0 for a specific value, and then continues the loop. If 0xFFFFFFF0 does not contain the specific value, CPU1 jumps to the fetched address. In this application note, CPU1 is not used so it continues running the wait for event loop indefinitely.

The CPU0 application repeats step 4 to step 7 indefinitely.

The MB0 application is located in memory starting at address 0x30000000. The linker script is used to set the starting address. The MB0 application performs the following:

1) Initializes the interrupt controller and interrupt subsystem.

2) Waits for a memory location in OCM that is used as a semaphore flag to be set.

3) Prints "MB0 : Waiting for IRQ" to the UART.

4) Waits for the interrupt service routine to increment the variable irq_count.

5) Waits for the UART TX FIFO to empty.

6) Clears the memory location in OCM that is used as a semaphore flag.

The MB0 application repeats step 2 to step 7 indefinitely

The inter-processor communication in the example design is a semaphore flag. When the semaphore is set, MB0 owns the UART, and when it is cleared by MB0, CPU0 is free to use the UART. This is a simple mechanism to share resources. The OCM memory is chosen because it is a low-latency, shared resource. Also, this area of OCM is not cached so the memory accesses are coherent and deterministic. The hardware setup configures the ZedBord to boot from the SD card. A terminal program should be configured to listen to the correct COM port with a baud rate of 115200 [14]. If the files were created correctly, CPU0 displays the message "CPU0: Hello World" followed by MB0 displaying "MB0 : Waiting for IRQ," as shown Figure 7. 


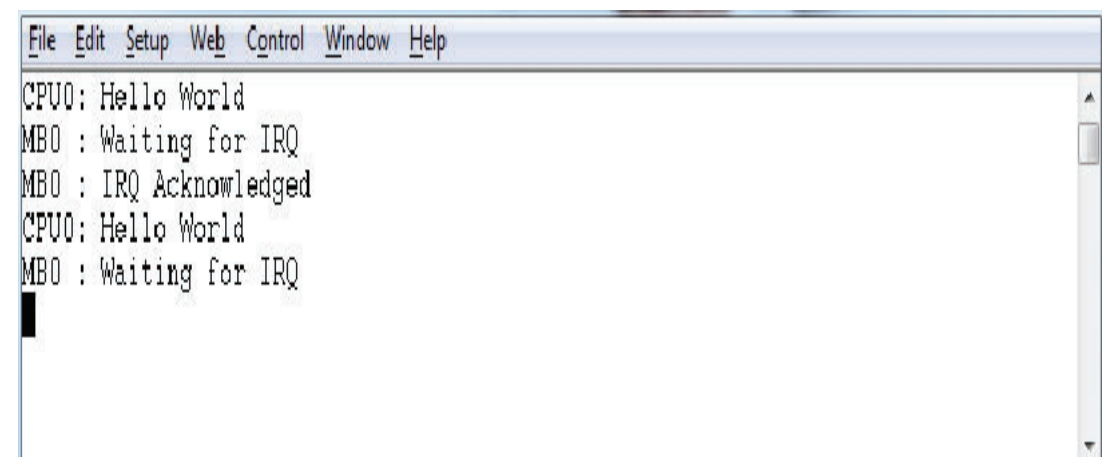

Figure 7. The Output Information Displaying in the Terminal

\section{Conclusion}

The design demonstrates how to boot the Zynq-7000 AP SoC and start a CortexA9 processor and MicroBlaze processor, each running their own bare-metal application. Leveraging the low overhead of a bare-metal application on MB0, an interrupt sourced from the PL is serviced and communicated to the bare-metal application running on CPU0. On-chip memory (OCM) is used as a shared memory for CPU0 and MB0, and the communication message is displayed. When compared to DDR memory, OCM provides very high performance and low latency access from both processors.

\section{Acknowledgments}

We would like to thank professor GE for stimulating discussions with respect to the topic of this paper and laboratory equipment. Moreover, we greatly appreciate the reviewers' comments that lead to an improved presentation of the results. This work was supported by Research Program of science and technology at Universities of Inner Mongolia Autonomous Region (NJZY282).

\section{References}

[1] Y. Nie, Z. Ma and L. Jing, "Research on the Design of Multi-Core Embedded System Based on Microblaze", International Journal of Control and Automation, vol. 8, no. 12, (2015), pp. 425 -434.

[2] A. Munir, A. Gordon-Ross and S. Ranka, "Multi-Core Embedded Wireless Sensor Networks: Architecture and Applications", IEEE Transactions on Parallel and Distributed Systems, vol. 25, no. 6, (2014), pp. 1553-1562.

[3] Xilinx, "Zynq-7000 All Programmable SoC Overview”, (2016).

[4] Xilinx, "Zynq-7000 All Programmable SoC Technical Reference Manual", (2015).

[5] Xilinx, "MicroBlaze Processor Reference Guide", (2015).

[6] M. Lee, M. Ahn and E. Jung Kim, "Fast Secure Communications in Shared Memory Multiprocessor Systems", IEEE Transactions on Parallel and Distributed Systems, vol. 22, no. 10, (2011), pp. 17141721.

[7] L. Yan, Q. Shi, T. Chen and G. Chen, "An On-chip Communication Mechanism Design in the Embedded Heterogeneous Multi-core Architecture", Proceedings of 2008 IEEE International Conference on Networking, Sensing and Control, Sanya, China, (2008) April 6-8.

[8] Xilinx, "LogiCORE IP Multi-Port Memory Controller (v6.06.a) Data Sheet", (2013).

[9] Xilinx, “AXI Reference Guide”, (2012).

[10] H. Kim and R. Bond, "Multi-core software technologies", IEEE Signal Processing Magazine, vol. 26, no. 6, (2009), pp. 80-89.

[11] X. Xu, N. Center and L. Wang, "Task assignments based on shared memory multi-core communication", Proceedings of the 2nd International Conference on Systems and Informatics, Shanghai, China, (2014) November 15-17.

[12] A. Yamawaki and M. Iwane, "An FPGA Implementation of a Snoop Cache With Synchronization for A Multiprocessor System-On-chip", Proceedings of the 13th International Conference on Parallel and Distributed Systems, Washington, DC, USA, (2007) December 5-7. 
[13] Y.-H. Lin, C. Tu, C.-S. Shih and S.-H. Hung, "Zero-Buffer Inter-core Process Communication Protocol for Heterogeneous Multi-core Platforms", Proceedings of the 15th IEEE International Conference on Embedded and Real-Time Computing Systems and Applications, Beijing, China, (2009) August 24-26.

[14] P. Fogarty, C. MacNamee and D. Heffernan, "On-chip support for software verification and debug in multi-core embedded systems", IET Software, vol. 7, no. 1, (2013), pp. 56 -64.

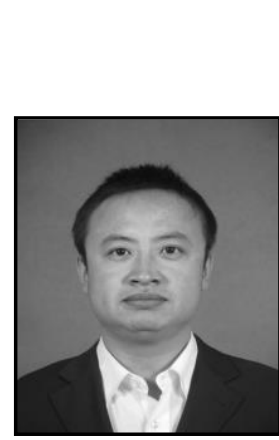

\begin{abstract}
Authors
Yang Nie, he received the MS degree from Wuhan Research Institute of Posts \&Telecommunications in 2009. He is currently working toward the $\mathrm{PhD}$ degree in the Digital Engineering Center of Communication University of China. He is also a lecturer of Jining Normal University, China. His research interest includes Compressive Sensing, High-performance DSP Algorithms and VLSI Architectures.
\end{abstract}

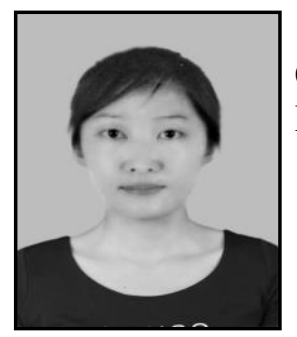

Lili Jing, she is currently a lecturer of Jining Normal University, China. Her research interest includes sensor detection technology, High-performance DSP Algorithms and VLSI Architectures.

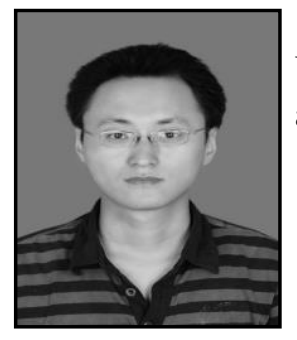

Pengyu Zhao, he is currently a lecturer of Jining Normal University, China. His research interest includes PLC system design, and program design using $\mathrm{C}++$. 